\title{
新型含酰基腿桥和硫胀桥的苯双酰胺类似物的合成及生物活性
}

\author{
陈有为 \\ 万莹莹 \\ 刘巧霞 \\ 刘敬波 \\ 熊丽霞 \\ 于淑晶李正名* \\ (南开大学元素有机化学研究所 元素有机化学国家重点实验室 天津化学化工协同创新中心 天津 300071)
}

\begin{abstract}
摘要 参考氟虫酰胺和除虫艮的活性结构片段, 设计合成了一系列结构新颖的含有酰基腿桥和酰基硫謜桥的化合物, 通过核磁共振氢谱、高分辨质谱以及 $\mathrm{X}$ 射线单晶衍射确定了其结构. 生测结果表明, 酰基腿桥和酰基硫腿桥结构的引 入使杀虫活性降低, 但是化合物 7d 在 $10 \mathrm{mg} / \mathrm{L}$ 的浓度下, 仍表现出一定的杀粘虫活性. 此外发现部分化合物具有一定 的抑菌活性，其中化合物 $8 \mathbf{8}$ 对苹果轮纹、小麦纹枯和水稻恶苗病原菌的抑制率分别为 $75.0 \%$ 、 $87.5 \%$ 和 $84.6 \%$.

关键词＼cjkstart氟虫酰胺; 除虫朖; 酰基腿桥; 硫腿桥; 杀虫活性; 抑菌活性
\end{abstract}

\section{Synthesis and Biological Activities of Novel Analogues of Phthalamide Containing Acylurea and Acylthiourea}

\author{
Chen, Youwei Wan, Yingying Liu, Qiaoxia Liu, Jingbo \\ Xiong, Lixia Yu, Shujing Li, Zhengming*
}

(State Key Laboratory of Elemento-organic Chemistry, Collaborative Innovation Center of Chemical Science and Engineering (Tianjin), Research Institute of Elemento-organic Chemistry, Nankai University, Tianjin 300071)

\begin{abstract}
In order to find novel and efficient insecticides targeting at the ryanodine receptor, a series of novel compounds containing acylurea and acylthiourea, referring to moieties of flubendiamide and diflubenzuron, were designed and synthesized. Their structures were characterized by ${ }^{1} \mathrm{H}$ NMR, HRMS and X-ray diffraction. The insectical activities showed that the introducing of acylurea and acylthiourea bridges decreased the acitivity. Compound $\mathbf{7 d}$ showed $50 \%$ activity at the concentration of $10 \mathrm{mg} / \mathrm{L}$. In addtion, some structures showed moderate antifungal activities. Compound 8f displayed $75.0 \%$, $87.5 \%$ and $84.6 \%$ antifungal activities against Botryosphaeria dothidea, Rhizoctonia cerealis and Fusarium fujikuroi.
\end{abstract}

Keywords flubendiamide; diflubenzuron; acylurea; acylthiourea; insecticidal activity; antifungal activity

创制具有高活性、高选择性、低毒和环境友好的农 药新品种是未来农药的发展趋势 ${ }^{[1 \sim 4]}$. 鱼尼丁受体 (RyR)通道是一类主要的 $\mathrm{Ca}^{2+}$ 释放通道, RyR 对细胞内 $\mathrm{Ca}^{2+}$ 浓度的平衡稳定起着十分重要的作用, 是一类新型 的杀虫剂作用靶标 ${ }^{[5 \sim 9]}$. 日本农药公司研发的氟虫酰胺 (Flubendiamide, 结构见图 1$)^{[10]}$ 是一类昆虫鱼尼丁受体 调节剂，是首类作用于昆虫 RyRs 的人工合成化合物. 它不仅与传统的杀虫剂无交互抗性, 而且对哺乳动物等 安全 ${ }^{[11,12]}$. 这类结构新颖、作用机制独特的新农药品种 引起了我们的关注 ${ }^{[13,14]}$.
除虫艮(Diflubenzuron, 结构见图 1)是 20 世纪 70 年 代发现的一类广谱、高效、低毒、高选择性的新型几丁

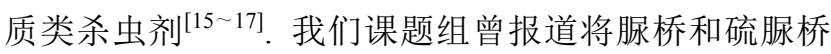
引入到杀虫剂氯虫酰胺(Rynaxypyr, DPX-E2Y45) ${ }^{[18]}$ 后, 所得到的化合物依然能保持很好的杀虫活性 ${ }^{[19]}$. 文献 中关于氟虫酰胺结构的改造大多保留了邻苯二甲酰胺 骨架结构 ${ }^{[20 ~ 21]}$, 而未见将䐂桥引入到氟虫酰胺骨架中 的报道. 本文参考除虫艮的结构将其苯基腿桥结构引入 到氟虫酰胺中(图 1)，替换原来的七氟异丙基的芳香胺， 设计了一系列新的结构, 以期望获得高活性化合物.

*E-mail: nkzml@vip.163.com

Received September 5, 2014; revised October 30, 2014; published online November 27, 2014.

Project supported by the National Basic Research Program of China (973 Program, No. 2010CB126106), the National Natural Science Foundation of China (Nos. 31370039, 21272129), the National Key Technologies R\&D Program (No. 2011BAE06B05), and the Specialized Research Fund for the Doctorial Program of Higher Education (No. 20110031110017).

国家重点基础研究发展计划(973 计划, No. 2010CB126106)、国家自然科学基金(Nos. 31370039, 21272129)、国家科技攻关计划(No. 2011BAE06B05)、 高等学校博士学科点专项科研基金(No. 20110031110017)资助项目. 


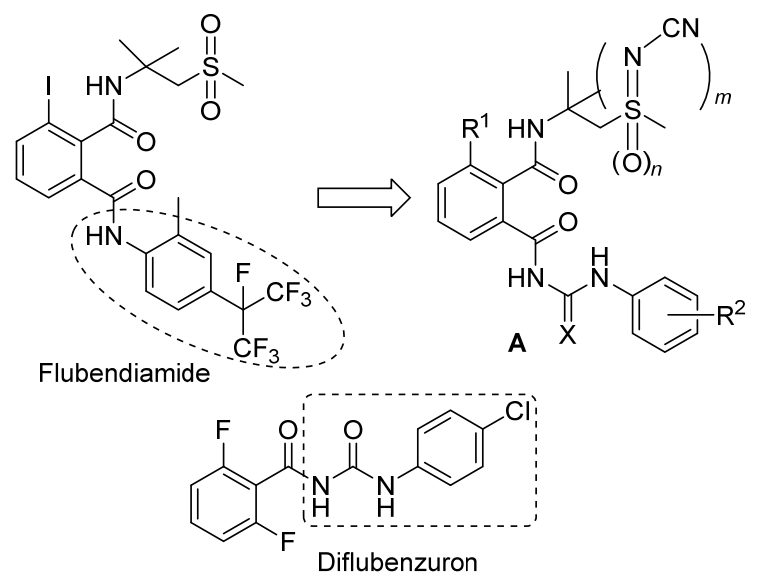

图 1 氟虫酰胺、除虫嫝和化合物 $\mathbf{A}$ 的结构

Figure 1 Chemical structures of flubendiamide, diflubenzuron and compound $\mathbf{A}$

\section{1 结果与讨论}

\section{1 合成讨论}

在中间体 2 (Scheme 1) 的生成过程中有副产物二苯
脲, 将反应液在沸腾状态下过滤, 滤饼大部分为二苯嫝, 滤液中几乎不含二苯脲, 滤液冷却中产生了大量固体, 用四氢呋喃(THF)重结晶得到纯的中间体.

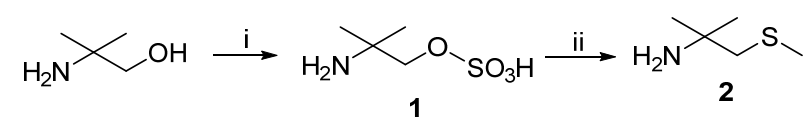

Reagents and conditions: (i) $\mathrm{ClSO}_{3} \mathrm{H}, \mathrm{Et}_{2} \mathrm{O},-10^{\circ} \mathrm{C}$; (ii) $\mathrm{NaSCH}_{3}$, $\mathrm{NaOH}, \mathrm{H}_{2} \mathrm{O}, 90^{\circ} \mathrm{C}$

图式 1 化合物 2 的合成路线

Scheme 1 Synthetic route of compound 2

在合成目标化合物 7 的过程中, 曾采用文献[19]中 的方法, 将酸 5 转化为酰氯, 然后与氨水或者硫氰酸钾 反应，最后与相应苯胺反应，但没有得到目标化合物， 可能由于酸 $\mathbf{5}$ 中含有酰胺键, 在加入草酰氯之后会发生 分子内合环从而无法得到所期的酰氯. 于是采用了 Scheme 2 中的方法, 成功得到结构 $7 \mathbf{a} \sim 7 \mathrm{~d}$. 在合成 $7 \mathbf{g}$ 的过程中, 收率非常低, 得到一个副产物 11. 通过对其 晶体结构分析, 发现是一类含有双硫键的结构, 可能的 机理在图 2 中进行描述.<smiles>[R]c1cccc2c1C(=O)OC2=O</smiles><smiles>CSCC(C)(C)N</smiles><smiles>[R]c1cccc(C(=O)O)c1C(=O)NC(C)(C)CSC</smiles>

$4 a \sim 6 a: R^{1}=N_{2} ; 4 b \sim 6 b: R^{1}=I$

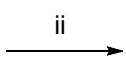<smiles>[R]c1cccc2c1C(=NC(C)(C)CSC)OC2=O</smiles><smiles>[R][X]c1cccc(NC(=O)NC(=O)c2cccc([R1])c2C(=O)NC(C)(C)CS(C)(=O)=O)c1</smiles>

8a: $\mathrm{R}^{1}=\mathrm{NO}_{2}, \mathrm{R}^{2}=\mathrm{H} ; \mathbf{8 b}: \mathrm{R}^{1}=\mathrm{I}, \mathrm{R}^{2}=\mathrm{H}$;

8c: $R^{1}=\mathrm{NO}_{2}, \mathrm{R}^{2}=4-\mathrm{Cl} ; 8 \mathrm{~d}: \mathrm{R}^{1}=\mathrm{I}, \mathrm{R}^{2}=4-\mathrm{Cl}$;

$8 e: R^{1}=I, R^{2}=4-F ; 8 f: R^{1}=I, R^{2}=3,4-F_{2}$<smiles>[R][X]1ccc(NC(=O)NC(=O)c2cccc([R1])c2C(=O)NC(C)(C)CS(C)=NC#N)cc1</smiles>

9a: $\mathrm{R}^{1}=\mathrm{NO}_{2}, \mathrm{R}^{2}=\mathrm{H} ; \mathbf{9 b}: \mathrm{R}^{1}=\mathrm{I}, \mathrm{R}^{2}=\mathrm{H}$;

9c: $\mathrm{R}^{1}=\mathrm{NO}_{2}, \mathrm{R}^{2}=4-\mathrm{Cl} ; 9 \mathrm{~d}: \mathrm{R}^{1}=\mathrm{I}, \mathrm{R}^{2}=4-\mathrm{Cl}$

Reagents and conditions: (i) $\mathrm{NH}_{2} \mathrm{C}\left(\mathrm{CH}_{3}\right)_{2} \mathrm{CH}_{2} \mathrm{SCH}_{3}, \mathrm{Et}_{3} \mathrm{~N}, \mathrm{CH}_{2} \mathrm{Cl}_{2}$, r.t.; (ii) $\mathrm{CH}_{3} \mathrm{SO}_{2} \mathrm{Cl}$, $\mathrm{Et}_{3} \mathrm{~N}$, $\mathrm{CH}_{2} \mathrm{Cl}_{2},-10{ }^{\circ} \mathrm{C}$; (iii) $\mathrm{CH}_{2} \mathrm{Cl}_{2},-10^{\circ} \mathrm{C} \sim$ r.t.; (iv) $m$-CPBA, THF, r.t.; (v) THF, $\mathrm{NH}_{2} \mathrm{CN}, \mathrm{PhIAc}_{2}$

图式 2 目标化合物 $7 \mathrm{a} \sim 7 \mathrm{~g}, 8 \mathrm{a} \sim 8 \mathrm{f}$ 和 $9 \mathrm{a} \sim 9 \mathrm{~d}$ 的合成路线

Scheme 2 Synthetic route of title compounds $7 \mathbf{a} \sim 7 \mathrm{~g}, 8 \mathrm{8} \sim 8 \mathrm{f}$ and $9 \mathrm{a} \sim 9 \mathrm{~d}$ 

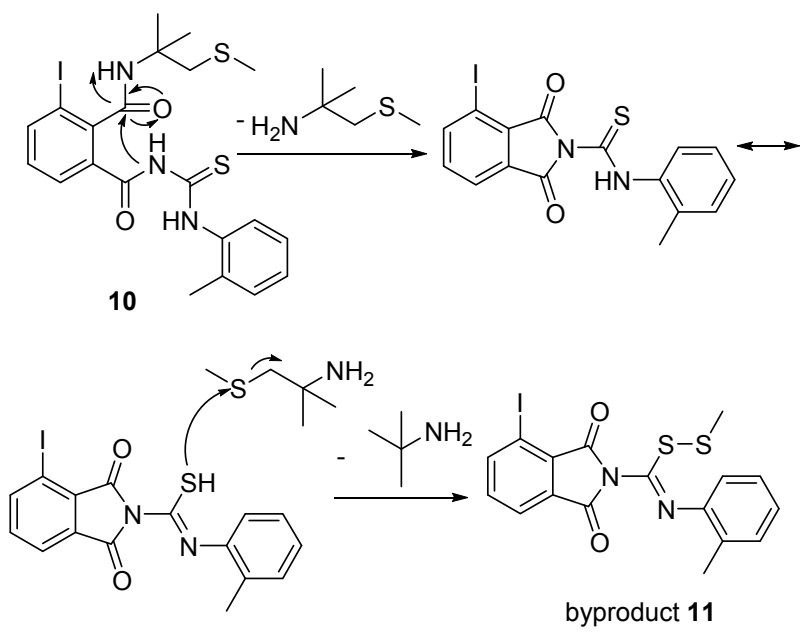

图 2 副产物 11 的合成机理推测

Figure 2 The synthesizing mechanism investigation of byproduct 11

\section{2 晶体结构}

晶体结构分析表明(图 3): 该分子 (CCDC 号为 1023049)含有一个过硫键, 键长为 $2.0306 \AA$. 苯环、过硫 键、邻苯二甲酰亚胺基、硝基的键长键角都与文献 [22 26]报道的一致. $\mathrm{C}(8)-\mathrm{N}(1)-\mathrm{C}(1)-\mathrm{O}(1) 、 \mathrm{C}(1)-$ $\mathrm{N}(1)-\mathrm{C}(8)-\mathrm{O}(2) 、 \mathrm{O}(1)-\mathrm{C}(1)-\mathrm{C}(2)-\mathrm{C}(7) 、 \mathrm{C}(2)-$ $\mathrm{C}(7)-\mathrm{C}(8)-\mathrm{O}(2)$ 之间二面角分别为 $-174.9(3)^{\circ}$ 、$179.4(4)^{\circ} 、 173.7(4)^{\circ} 、 177.0(4)^{\circ}$, 全部几乎共面, 说明了 邻苯二甲酰亚胺基中的羰基与氮原子和苯环存在很强 的共轭作用. 邻苯二甲酰亚胺基的五元环 $\mathrm{C}(1)-\mathrm{C}(2)-$ $\mathrm{C}(7)-\mathrm{C}(8)-\mathrm{N}(1)$ 为一个平面, 与苯环 $\mathrm{C}(2)-\mathrm{C}(3)-$ $C(4)-C(5)-C(6)-C(7)$ 的二面角为 $1.6^{\circ}$, 几乎共面, 再 次证明羰基与苯环之间的共轭作用. 苯环 $\mathrm{C}(10)$ $\mathrm{C}(11)-\mathrm{C}(12)-\mathrm{C}(13)-\mathrm{C}(14)-\mathrm{C}(15)$ 与苯环 $\mathrm{C}(2)-$ $\mathrm{C}(3)-\mathrm{C}(4)-\mathrm{C}(5)-\mathrm{C}(6)-\mathrm{C}(7)$ 的二面角为 $5.4^{\circ}$, 基本在 同一平面.

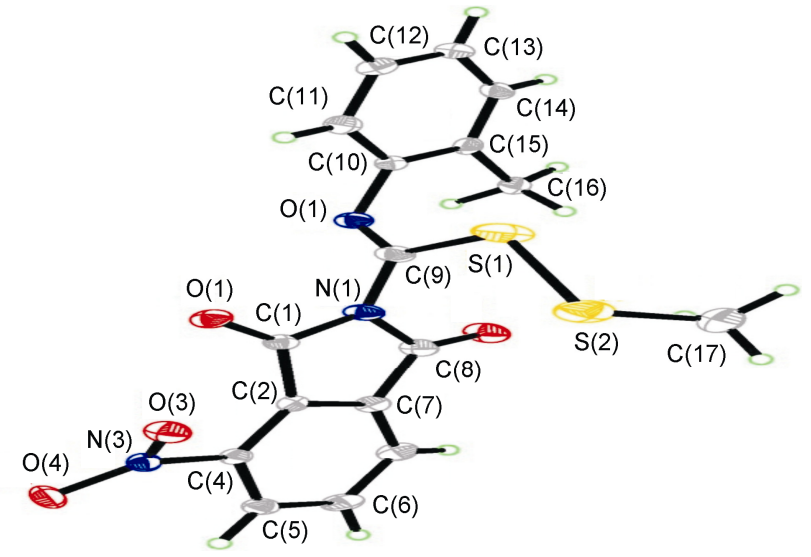

图 3 化合物 $\mathbf{1 1}$ 的晶体结构

Figure 3 Crystal structure of compound 11

\section{3 生物活性}

目标化合物 7a $\sim 7 g, 8 a \sim 8 f$ 和 9a $\sim 9 d$ 对东方粘虫 (Mythimna separata) 的生物活性测试结果见表 1 , 大部 分目标化合物对东方粘虫的杀虫活性一般, $7 \mathbf{b}$ 和 7d 在 $50 \mathrm{mg} / \mathrm{L}$ 的浓度下分别表现出 $50 \%$ 和 $100 \%$ 的杀虫活性. 当用除虫嫝中的 4 -氯苯基脲桥结构替换氟虫酰胺分子 中含有七氟异丙基的芳香胺之后，化合物 7d 在 $10 \mathrm{mg} / \mathrm{L}$ 的浓度下表现出 $50 \%$ 的杀虫活性, 活性有一定程度降 低. 这可能是由如下原因所导致, 首先酰胺桥变为脲桥 后, 化合物不能很好地与受体进行结合, 其次七氟异丙 基较大的空间位阻、高电负性以及氟原子本身的特性对 活性的保持起到了很大的作用.

对目标化合物的抑菌活性测试结果见表 2 , 在 50 $\mathrm{mg} / \mathrm{L}$ 的浓度下, 目标化合物对 12 种菌均表现出一定的 抑制活性. 大部分化合物对油菜菌核, 苹果轮纹和小麦 纹枯都表现出中等的抑菌活性. 化合物 $7 \mathrm{~b}$ 和 $9 \mathrm{c}$ 对油菜 菌核的抑制率分别为 $60.9 \%$ 和 $71.1 \%$. 化合物 $8 \mathbf{f}$ 对苹果 轮纹、小麦纹枯和水稻恶苗表现出 $75.0 \%, 87.5 \%, 84.6 \%$ 的抑菌活性, 化合物 $8 \mathrm{~b}$ 和 $9 \mathrm{~b}$ 分别对小麦纹枯表现出 $72.3 \%$ 和 $66.3 \%$ 的抑菌活性. $8 \mathrm{~b}$ 对大部分菌类都表现出 中等或较高的抑菌活性, 尤其对苹果轮纹和小麦纹枯分 别表现出 $65.0 \%$ 和 $72.3 \%$ 的抑制率. 因此, 该结构可以 进一步优化，以寻找抑菌活性更高的化合物.

\section{2 结论}

本文设计合成了一系列含有腿桥和硫脲桥的氟虫 酰胺类似物, 生测结果表明, 大部分目标化合物对东方 粘虫的杀虫活性一般, 但是化合物 7b 和 7d 表现出较好 的杀粘虫活性, 7d 在 $10 \mathrm{mg} / \mathrm{L}$ 的浓度下, 表现出 $50 \%$ 的 杀虫活性. 该结构有待于进一步优化. 还发现该系列化 合物表现出一定的抑菌活性. 化合物 $8 \mathrm{f}$ 对苹果轮纹、小 麦纹枯和水稻恶苗表现出 $75.0 \%, 87.5 \%, 84.6 \%$ 的抑菌 活性, 化合物 $8 \mathrm{~b}$ 对大部分真菌表现出较明显的抑菌活 性, 这两个结构可作为先导化合物来寻找抑菌活性更好 的结构.

\section{3 试验部分}

\section{1 仪器与试剂}

X-4 数字显示显微熔点测定仪(北京泰克仪器有限 公司); FTICR-MS (Varian 7.0T) 型高分辨率质谱仪; Bruker Avance- $300 \mathrm{MHz}$ 和 Bruker Avance- $400 \mathrm{MHz}$ 核 磁共振仪, $\mathrm{CDCl}_{3}$ 或 DMSO- $d_{6}$ 为溶剂, TMS 为内标. 所 用试剂均为市售分析纯或化学纯. 
表 1 目标化合物和氟虫酰胺对东方粘虫的致死率

Table 1 Insecticidal activities of title compounds and flubendiamide against Oriental armywarms<smiles>[R]c1ccc(NC([X])NC(=O)c2cccc([R])c2C(=O)NC(C)(C)C[SH](C)(C)=O)cc1</smiles>

\begin{tabular}{|c|c|c|c|c|c|c|c|c|c|c|}
\hline \multirow{2}{*}{ Compd. } & \multirow{2}{*}{$\mathrm{R}^{1}$} & \multirow{2}{*}{$\mathrm{R}^{2}$} & \multirow{2}{*}{$\mathrm{X}$} & \multirow{2}{*}{$m$} & \multirow{2}{*}{$n$} & \multicolumn{5}{|c|}{ Larvicidal activity $/ \%$} \\
\hline & & & & & & $200 \mathrm{mg} / \mathrm{L}$ & $100 \mathrm{mg} / \mathrm{L}$ & $50 \mathrm{mg} / \mathrm{L}$ & $25 \mathrm{mg} / \mathrm{L}$ & $10 \mathrm{mg} / \mathrm{L}$ \\
\hline $7 a$ & $\mathrm{NO}_{2}$ & $\mathrm{H}$ & $\mathrm{O}$ & 0 & 0 & 40 & & & & \\
\hline $7 b$ & I & $\mathrm{H}$ & $\mathrm{O}$ & 0 & 0 & 100 & 100 & 50 & & \\
\hline $7 c$ & $\mathrm{NO}_{2}$ & $4-\mathrm{Cl}$ & $\mathrm{O}$ & 0 & 0 & 50 & & & & \\
\hline $7 d$ & I & $4-\mathrm{Cl}$ & $\mathrm{O}$ & 0 & 0 & 100 & 100 & 100 & 70 & 50 \\
\hline $7 e$ & I & $4-\mathrm{F}$ & $\mathrm{O}$ & 0 & 0 & 20 & & & & \\
\hline $7 f$ & I & $3,4-\mathrm{F}_{2}$ & $\mathrm{O}$ & 0 & 0 & 60 & & & & \\
\hline $7 \mathrm{~g}$ & $\mathrm{NO}_{2}$ & $2-\mathrm{CH}_{3}$ & $\mathrm{~S}$ & 0 & 0 & 60 & & & & \\
\hline $8 a$ & $\mathrm{NO}_{2}$ & $\mathrm{H}$ & $\mathrm{O}$ & 0 & 2 & 20 & & & & \\
\hline $8 b$ & I & $\mathrm{H}$ & $\mathrm{O}$ & 0 & 2 & 20 & & & & \\
\hline $8 c$ & $\mathrm{NO}_{2}$ & $4-\mathrm{Cl}$ & $\mathrm{O}$ & 0 & 2 & 0 & & & & \\
\hline $8 d$ & I & $4-\mathrm{Cl}$ & $\mathrm{O}$ & 0 & 2 & 60 & & & & \\
\hline $8 e$ & I & $4-\mathrm{F}$ & $\mathrm{O}$ & 0 & 2 & 40 & & & & \\
\hline $8 f$ & I & $3,4-\mathrm{F}_{2}$ & $\mathrm{O}$ & 0 & 2 & 80 & & & & \\
\hline $9 a$ & $\mathrm{NO}_{2}$ & $\mathrm{H}$ & $\mathrm{O}$ & 1 & 0 & 0 & & & & \\
\hline $9 b$ & I & $\mathrm{H}$ & $\mathrm{O}$ & 1 & 0 & 30 & & & & \\
\hline $9 c$ & $\mathrm{NO}_{2}$ & $4-\mathrm{Cl}$ & $\mathrm{O}$ & 1 & 0 & 0 & & & & \\
\hline 9d & I & $4-\mathrm{Cl}$ & $\mathrm{O}$ & 1 & 0 & 10 & & & & \\
\hline Flubendiamide & & & & & & 100 & 100 & 100 & 100 & 100 \\
\hline
\end{tabular}

表 2 目标化合物在 $50 \mathrm{mg} / \mathrm{L}$ 剂量下的抑菌活性 ${ }^{a}$

Table 2 The fungicidal activity of title compounds at $50 \mathrm{mg} / \mathrm{L}$

\begin{tabular}{ccccccccccccc}
\hline Compd. & $\mathrm{A}$ & $\mathrm{B}$ & $\mathrm{C}$ & $\mathrm{D}$ & $\mathrm{E}$ & $\mathrm{F}$ & $\mathrm{G}$ & $\mathrm{H}$ & $\mathrm{I}$ & $\mathrm{J}$ & $\mathrm{K}$ & $\mathrm{L}$ \\
\hline $\mathbf{7 a}$ & 22.7 & 27.3 & 15.6 & 32.6 & 10.3 & 14.0 & 21.4 & 06.7 & 24.1 & 12.5 & 13.5 & 14.3 \\
$\mathbf{7 b}$ & 18.2 & 21.2 & 21.9 & 60.9 & 20.6 & 16.3 & 21.4 & 16.7 & 49.4 & 27.5 & 18.9 & 25.0 \\
$\mathbf{7 c}$ & 13.6 & 48.5 & 18.8 & 54.3 & 17.6 & 23.3 & 25.0 & 31.7 & 38.6 & 17.5 & 18.9 & 17.9 \\
$\mathbf{7 d}$ & 13.6 & 24.2 & 28.1 & 41.3 & 32.4 & 30.2 & 25.0 & 65.0 & 61.4 & 22.5 & 29.7 & 25.0 \\
$\mathbf{7 e}$ & 11.1 & 22.2 & 16.1 & 12.5 & 10.0 & 25.0 & 36.4 & 31.3 & 62.5 & 33.3 & 26.7 & 46.2 \\
$\mathbf{7 f}$ & 11.1 & 11.1 & 11.3 & 16.1 & 10.0 & 31.3 & 36.4 & 31.3 & 62.5 & 38.9 & 26.7 & 53.8 \\
$\mathbf{8 a}$ & 45.5 & 12.1 & 15.6 & 43.5 & 20.6 & 9.3 & 7.1 & 28.3 & 22.9 & 12.5 & 8.1 & 7.1 \\
$\mathbf{8 b}$ & 50.0 & 54.5 & 40.6 & 43.5 & 45.6 & 34.9 & 53.6 & 65.0 & 72.3 & 47.5 & 56.8 & 25.0 \\
$\mathbf{8 c}$ & 13.6 & 12.1 & 34.4 & 21.7 & 33.8 & 14.0 & 14.3 & 21.7 & 54.2 & 20.0 & 27.0 & 17.9 \\
$\mathbf{8 d}$ & 40.9 & 12.1 & 25.0 & 21.7 & 42.6 & 23.3 & 35.7 & 55.0 & 50.6 & 30.0 & 29.7 & 10.7 \\
$\mathbf{8 e}$ & 16.7 & 13.9 & 12.9 & 10.7 & 10.0 & 25.0 & 36.4 & 37.5 & 68.8 & 33.3 & 33.3 & 46.2 \\
$\mathbf{8 f}$ & 11.1 & 11.1 & 11.3 & 10.7 & 11.3 & 31.3 & 45.5 & 75.0 & 87.5 & 44.4 & 40.0 & 84.6 \\
$\mathbf{9 a}$ & 13.6 & 15.2 & 28.1 & 39.1 & 29.4 & 9.3 & 0.0 & 36.7 & 16.9 & 17.5 & 2.7 & 3.6 \\
$\mathbf{9 b}$ & 22.7 & 12.1 & 34.4 & 43.5 & 19.1 & 27.9 & 25.0 & 65.0 & 66.3 & 15.0 & 21.6 & 3.6 \\
$\mathbf{9 c}$ & 45.5 & 36.4 & 37.5 & 71.1 & 36.8 & 20.9 & 39.3 & 43.3 & 55.4 & 42.5 & 37.8 & 32.1 \\
$\mathbf{9 d}$ & 27.3 & 12.1 & 18.8 & 52.2 & 13.2 & 2.3 & 17.9 & 40.0 & 13.1 & 12.5 & 10.8 & 10.7 \\
百菌清 & 73.9 & 73.1 & 82.6 & 96.4 & 96.1 & 100 & 75.0 & 92.3 & 100 & 90.4 & 95.0 & 100 \\
\hline A
\end{tabular}

${ }^{a} \mathrm{~A}$ : 番茄早疫(Alternaria solani Sorauer), B: 小麦赤霉(Fusarium graminearum), C: 辣椒疫霉(Phytophthora capsici), D: 油菜菌核(Sclerotinia sclerotiorum), E: 水稻纹枯(Rhizoctonia solani), F: 黄瓜枯萎(Cucumber Fusarium Wilt), G: 花生褐斑(Cercospora arachidicola), H: 苹果轮纹(Botryosphaeria dothidea), I: 小麦 纹枯(Rhizoctonia cerealis), J: 玉米小斑(Helminthosporium maydis), K: 西瓜炭疽(Colletotrichum lagenarium), L: 水稻恶苗(Fusarium moniliforme sheld). 


\subsection{1,1-二甲基-2-甲硫基乙胺(2)的合成}

中间体 2 的合成参考文献[30]. 化合物 2: 无色液 体, 产率 76\%. b.p. $155 \sim 156{ }^{\circ} \mathrm{C} ;{ }^{1} \mathrm{H}$ NMR $(400 \mathrm{~Hz}$, $\left.\mathrm{CDCl}_{3}\right) \delta: 1.182\left(\mathrm{~s}, 6 \mathrm{H}, \mathrm{C}\left(\mathrm{CH}_{3}\right)_{2}\right), 2.179\left(\mathrm{~s}, 3 \mathrm{H}, \mathrm{SCH}_{3}\right)$, $2.574\left(\mathrm{~s}, 2 \mathrm{H}, \mathrm{CH}_{2} \mathrm{SCH}_{3}\right), 4.839\left(\mathrm{~s}, 2 \mathrm{H}, \mathrm{NH}_{2}\right)$.

\section{3 苯基艮 $3 a \sim 3 d$ 的合成}

中间体 3a 3d 的合成参考文献[27].

化合物 3a: 白色固体, 产率 78\%. m.p. 147 $148{ }^{\circ} \mathrm{C} ;{ }^{1} \mathrm{H}$ NMR $\left(400 \mathrm{~Hz}, \mathrm{CDCl}_{3}\right) \delta: 4.669$ (s, 2H, $\mathrm{NH}_{2}$ ), $6.449(\mathrm{~s}, 1 \mathrm{H}, \operatorname{ArNH}), 7.148(\mathrm{t}, J=6.8 \mathrm{~Hz}, 1 \mathrm{H}, \operatorname{ArH})$, $7.294 \sim 7.369(\mathrm{~m}, 4 \mathrm{H}, \mathrm{ArH})$.

化合物 3b: 白色固体, 产率 80\%. m.p. 209 $210{ }^{\circ} \mathrm{C} ;{ }^{1} \mathrm{H}$ NMR $\left(400 \mathrm{~Hz}, \mathrm{DMSO}-d_{6}\right) \delta: 5.904(\mathrm{~s}, 2 \mathrm{H}$, $\mathrm{NH}_{2}$ ), 7.249 (d, $\left.J=8.4 \mathrm{~Hz}, 2 \mathrm{H}, \mathrm{ArH}\right), 7.420$ (d, $J=8.8 \mathrm{~Hz}$, $2 \mathrm{H}, \mathrm{ArH}), 8.661$ (s, 1H, ArNH).

化合物 3c: 白色固体, 产率 76\%. m.p. 130 $131{ }^{\circ} \mathrm{C} ;{ }^{1} \mathrm{H}$ NMR (400 Hz, DMSO- $\left.d_{6}\right) \delta:{ }^{1} \mathrm{H}$ NMR (400 $\left.\mathrm{Hz}, \mathrm{DMSO}-d_{6}\right) \delta: 5.837\left(\mathrm{~s}, 2 \mathrm{H}, \mathrm{NH}_{2}\right), 7.048$ (t, $J=8.8 \mathrm{~Hz}$, 2H, ArH), 7.391 (dd, $J=8.8,5.0 \mathrm{~Hz}, 2 \mathrm{H}, \mathrm{ArH}), 8.564$ (s, $1 \mathrm{H}, \mathrm{ArNH})$.

化合物 3d: 白色固体，产率 76\%. m.p. 124 $125{ }^{\circ} \mathrm{C} ;{ }^{1} \mathrm{H}$ NMR $\left(400 \mathrm{~Hz}\right.$, DMSO- $\left.d_{6}\right) \delta:{ }^{1} \mathrm{H}$ NMR (400 $\left.\mathrm{Hz}, \mathrm{DMSO}-d_{6}\right) \delta: 5.962\left(\mathrm{~s}, 2 \mathrm{H}, \mathrm{NH}_{2}\right), 7.012(\mathrm{t}, J=8.8 \mathrm{~Hz}$, 1H, ArH), 7.263 (q, $J=9.3 \mathrm{~Hz}, 1 \mathrm{H}, \mathrm{ArH}), 7.637$ (ddd, $J=$ 13.8, 7.5, $25 \mathrm{~Hz}, 1 \mathrm{H}, \mathrm{ArH}), 8.772$ (s, 1H, ArNH).

\subsection{2-甲基苯基硫嫝(3e)的合成}

中间体 $3 \mathbf{e}$ 的合成参考文献[28, 29]. 化合物 3e: 白 色固体, 产率 83\%. m.p. 161 $162{ }^{\circ} \mathrm{C}$; ${ }^{1} \mathrm{H}$ NMR $(400 \mathrm{~Hz}$, $\left.\mathrm{CDCl}_{3}\right) \delta: 2.328\left(\mathrm{~s}, 3 \mathrm{H}, \mathrm{CH}_{3}\right), 6.080\left(\mathrm{~s}, 2 \mathrm{H}, \mathrm{NH}_{2}\right), 7.278$ (m, 4H, ArH), 8.175 (s, 1H, ArNH).

\section{5 化合物 5 的合成}

化合物 5 的合成参考文献[30], 化合物 $\mathbf{5 a}$ 和 $\mathbf{5 b}$ 的 核磁以及熔点数据和文献中一致.

\section{6 目标化合物 $7 a \sim 7 g, 8 a \sim 8 f$ 和 $9 a \sim 9 d$ 的合成}

\subsection{1 目标化合物 7 的合成}

目标化合物 $7 \mathbf{a} \sim 7 \mathbf{g}$ 的合成参考文献[31，32]. 冰盐 浴下, 将化合物 5 (3 mmol)溶解到 $20 \mathrm{~mL}$ 二氯甲烷 $\left(\mathrm{CH}_{2} \mathrm{Cl}_{2}\right)$ 中, 依次加入三乙胺(3.6 mmmol)和甲基黄酰氯 (3.6 mmol), 冰盐浴下搅拌 $0.5 \sim 1 \mathrm{~h}$, 得到含有化合物 6 的反应液, 不加处理, 直接向其加入化合物 $\mathbf{3}$, 冰浴下 摚拌 $1 \mathrm{~h}$, 缓慢升温至室温. 室温搅拌过夜, 次日回流 8 $\mathrm{h}$, 点板跟踪反应. 待反应结束后, 将溶剂旋干, 用 1 $\mathrm{mol} / \mathrm{L}$ 稀盐酸、饱和 $\mathrm{NaHCO}_{3}$ 溶液、饱和 $\mathrm{NaCl}$ 溶液洗
涤, 并用乙酸乙酯萃取. 合并有机相, 脱去溶剂, 残余 物经柱层析纯化得到化合物 7.

$N^{2}$-(2-甲基-1-甲硫基-2-丙基)- $N^{1}$-苯嫝基-3-硝基邻 苯二甲酰胺 $(7 \mathbf{a})$ : 白色固体，产率 65\%. m.p. 185 $186{ }^{\circ} \mathrm{C} ;{ }^{1} \mathrm{H}$ NMR $\left(400 \mathrm{~Hz}, \mathrm{CDCl}_{3}\right) \delta: 1.535[\mathrm{~s}, 6 \mathrm{H}$, $\left.\mathrm{C}\left(\mathrm{CH}_{3}\right)_{2}\right], 2.070\left(\mathrm{~s}, 3 \mathrm{H}, \mathrm{SCH}_{3}\right), 2.938\left(\mathrm{~s}, 2 \mathrm{H}, \mathrm{CH}_{2} \mathrm{~S}\right), 6.164$ [s, $\left.1 \mathrm{H}, \mathrm{CONHC}\left(\mathrm{CH}_{3}\right)_{2}\right], 7.147$ (t, $\left.J=7.2 \mathrm{~Hz}, 1 \mathrm{H}, \mathrm{ArH}\right)$, 7.334 (t, $J=8.0 \mathrm{~Hz}, 2 \mathrm{H}, \mathrm{ArH}), 7.470$ (d, $J=8.0 \mathrm{~Hz}, 2 \mathrm{H}$, ArH), 7.680 (d, $J=8.0 \mathrm{~Hz}, 1 \mathrm{H}, \mathrm{ArH}), 8.008$ (d, $J=8.0 \mathrm{~Hz}$, 1H, ArH), 8.268 (d, J=8.0 Hz, 1H, ArH), 9.497 (s, 1H, ArNH), 10.420 (s, 1H, CONHCO); ESI-FTICR-MS calcd for $\mathrm{C}_{20} \mathrm{H}_{23} \mathrm{~N}_{4} \mathrm{O}_{5} \mathrm{~S}[\mathrm{M}+\mathrm{H}]^{+}:$431.1389, found 431.1383.

$N^{2}$-(2-甲基-1-甲硫基-2-丙基)- $N^{1}$-苯脲基-3-碘邻苯 二甲酰胺(7b): 白色固体，产率 56\%. m.p. 200 201 ${ }^{\circ} \mathrm{C}$; ${ }^{1} \mathrm{H}$ NMR $\left(400 \mathrm{~Hz}, \mathrm{CDCl}_{3}\right) \delta: 1.502\left[\mathrm{~s}, 6 \mathrm{H}, \mathrm{C}\left(\mathrm{CH}_{3}\right)_{2}\right], 2.052$ $\left(\mathrm{s}, 3 \mathrm{H}, \mathrm{SCH}_{3}\right), 2.929\left(\mathrm{~s}, 2 \mathrm{H}, \mathrm{CH}_{2} \mathrm{~S}\right), 6.405[\mathrm{~s}, 1 \mathrm{H}$, $\left.\mathrm{CONHC}\left(\mathrm{CH}_{3}\right)_{2}\right], 7.142$ (t, $\left.J=7.6 \mathrm{~Hz}, 1 \mathrm{H}, \mathrm{ArH}\right), 7.320(\mathrm{t}$, $J=8.0 \mathrm{~Hz}, 2 \mathrm{H}, \mathrm{ArH}), 7.432$ (d, $J=8.0 \mathrm{~Hz}, 2 \mathrm{H}, \mathrm{ArH})$, 7.594 (t, $J=8.0 \mathrm{~Hz}, 1 \mathrm{H}, \mathrm{ArH}), 7.934$ (d, $J=7.6 \mathrm{~Hz}, 1 \mathrm{H}$, ArH), 8.182 (d, $J=8.4 \mathrm{~Hz}, 1 \mathrm{H}, \operatorname{ArH}), 10.057$ (s, 1H, ArNH), 10.433 (s, 1H, CONHCO); ESI-FTICR-MS calcd for $\mathrm{C}_{20} \mathrm{H}_{23} \mathrm{IN}_{3} \mathrm{O}_{3} \mathrm{~S}[\mathrm{M}+\mathrm{H}]^{+}: 512.0505$, found 512.0493 .

$N^{2}$-(2-甲基-1-甲硫基-2-丙基)- $N^{1}$-(4-氯苯嫝基)-3-硝 基邻苯二甲酰胺 $(7 \mathrm{c})$ ：白色固体，产率 70\%. m.p. 165 $166{ }^{\circ} \mathrm{C} ;{ }^{1} \mathrm{H}$ NMR $(400 \mathrm{~Hz}$, DMSO-d $) \delta: 1.347[\mathrm{~s}, 6 \mathrm{H}$, $\left.\mathrm{C}\left(\mathrm{CH}_{3}\right)_{2}\right], 2.053\left(\mathrm{~s}, 3 \mathrm{H}, \mathrm{SCH}_{3}\right), 2.908\left(\mathrm{~s}, 2 \mathrm{H}, \mathrm{CH}_{2} \mathrm{~S}\right), 7.427$ (d, $J=8.8 \mathrm{~Hz}, 2 \mathrm{H}, \mathrm{ArH}), 7.590$ (d, $J=8.4 \mathrm{~Hz}, 2 \mathrm{H}, \mathrm{ArH})$, 7.756 (t, $J=7.6 \mathrm{~Hz}, 1 \mathrm{H}, \operatorname{ArH}), 7.978$ (d, $J=7.6 \mathrm{~Hz}, 1 \mathrm{H}$, ArH), 8.211 (d, $J=8.4 \mathrm{~Hz}, 1 \mathrm{H}, \operatorname{ArH}), 8.316[\mathrm{~s}, 1 \mathrm{H}$, $\left.\mathrm{CONHC}\left(\mathrm{CH}_{3}\right)_{2}\right], 10.509$ (s, 1H, ArNH), 11.103 (s, 1H, CONHCO); ESI-FTICR-MS calcd for $\mathrm{C}_{20} \mathrm{H}_{22} \mathrm{ClN}_{4} \mathrm{O}_{5} \mathrm{~S}$ $[\mathrm{M}+\mathrm{H}]^{+}:$465.0999, found 465.0990.

$N^{2}$-(2-甲基-1-甲硫基-2-丙基)- $N^{1}$-(4-氯苯脲基)-3-碘 邻苯二甲酰胺 $(7 d)$ : 白色固体，产率 $67 \%$. m.p. 157 $158{ }^{\circ} \mathrm{C} ;{ }^{1} \mathrm{H}$ NMR $\left(400 \mathrm{~Hz}, \mathrm{CDCl}_{3}\right) \delta: 1.590[\mathrm{~s}, 6 \mathrm{H}$, $\left.\mathrm{C}\left(\mathrm{CH}_{3}\right)_{2}\right], 2.118\left(\mathrm{~s}, 3 \mathrm{H}, \mathrm{SCH}_{3}\right), 3.014\left(\mathrm{~s}, 2 \mathrm{H}, \mathrm{SCH}_{2}\right), 6.027$ $\left[\mathrm{s}, 1 \mathrm{H}, \mathrm{CONHC}\left(\mathrm{CH}_{3}\right)_{2}\right], 7.209(\mathrm{t}, J=8.0 \mathrm{~Hz}, 1 \mathrm{H}, \mathrm{ArH})$, $7.302(\mathrm{~d}, J=8.8 \mathrm{~Hz}, 2 \mathrm{H}, \mathrm{ArH}), 7.474(\mathrm{~d}, J=8.8 \mathrm{~Hz}, 2 \mathrm{H}$, $\operatorname{ArH}), 7.734$ (d, $J=7.6 \mathrm{~Hz}, 1 \mathrm{H}, \operatorname{ArH}), 8.036$ (d, $J=8.0 \mathrm{~Hz}$, $1 \mathrm{H}, \mathrm{ArH}), 9.351$ (s, 1H, ArNH), 10.581 (s, 1H, CONHCO); ESI-FTICR-MS calcd for $\mathrm{C}_{20} \mathrm{H}_{22} \mathrm{ClIN}_{3} \mathrm{O}_{3} \mathrm{~S}$ $[\mathrm{M}+\mathrm{H}]^{+}:$546.0115, found 546.0102.

$N^{2}$-(2-甲基-1-甲硫基-2-丙基)- $N^{1}$-(4-氟苯脲基)-3-碘 邻苯二甲酰胺 $(7 \mathrm{e})$ : 白色固体，产率 $75 \%$. m.p. 93 $94{ }^{\circ} \mathrm{C}$; ${ }^{1} \mathrm{H}$ NMR (400 Hz, DMSO- $\left.d_{6}\right) \delta$ : $1.382[\mathrm{~s}, 6 \mathrm{H}$, 
$\left.\mathrm{C}\left(\mathrm{CH}_{3}\right)_{2}\right], 2.055\left(\mathrm{~s}, 3 \mathrm{H}, \mathrm{SCH}_{3}\right), 2.958\left(\mathrm{~s}, 2 \mathrm{H}, \mathrm{CH}_{2} \mathrm{~S}\right), 7.204$ (t, $J=8.0 \mathrm{~Hz}, 2 \mathrm{H}, \mathrm{ArH}), 7.243(\mathrm{t}, J=8.4 \mathrm{~Hz}, 1 \mathrm{H}, \mathrm{ArH})$, $7.545 \sim 7.580(\mathrm{~m}, 2 \mathrm{H}, \mathrm{ArH}), 7.643(\mathrm{~d}, J=7.6 \mathrm{~Hz}, 1 \mathrm{H}$, ArH), $8.026(\mathrm{~d}, J=7.2 \mathrm{~Hz}, 1 \mathrm{H}, \operatorname{ArH}), 8.069[\mathrm{~s}, 1 \mathrm{H}$, $\left.\mathrm{CONHC}\left(\mathrm{CH}_{3}\right)_{2}\right], 10.514$ (s, 1H, ArNH), 10.714 (s, $1 \mathrm{H}$, CONHCO); ESI-FTICR-MS calcd for $\mathrm{C}_{20} \mathrm{H}_{22} \mathrm{FIN}_{3} \mathrm{O}_{3} \mathrm{~S}$ $[\mathrm{M}+\mathrm{H}]^{+}:$530.0411, found 530.0402 .

$N^{2}$-(2-甲基-1- 甲硫基-2-丙基)- $N^{1}$-(3,4-二氟苯艮 基)-3-碘邻苯二甲酰胺(7f): 白色固体, 产率 72\%. m.p. 96 97 ${ }^{\circ} \mathrm{C} ;{ }^{1} \mathrm{H}$ NMR $\left(400 \mathrm{~Hz}, \mathrm{CDCl}_{3}\right) \delta: 1.570[\mathrm{~s}, 6 \mathrm{H}$, $\left.\mathrm{C}\left(\mathrm{CH}_{3}\right)_{2}\right], 2.112$ (s, 3H, $\left.\mathrm{SCH}_{3}\right), 2.999$ (s, $\left.2 \mathrm{H}, \mathrm{CH}_{2} \mathrm{~S}\right), 6.074$ [s, $\left.1 \mathrm{H}, \mathrm{CONHC}\left(\mathrm{CH}_{3}\right)_{2}\right], 7.073(\mathrm{~d}, J=3.2 \mathrm{~Hz}, 1 \mathrm{H}, \mathrm{ArH})$, 7.110 (d, $J=9.4 \mathrm{~Hz}, 1 \mathrm{H}, \mathrm{ArH}), 7.189$ (t, $J=7.9 \mathrm{~Hz}, 1 \mathrm{H}$, ArH), $7.550 \sim 7.603(\mathrm{~m}, 1 \mathrm{H}, \mathrm{ArH}), 7.695$ (d, $J=7.0 \mathrm{~Hz}$, 1H, ArH), 7.997 (d, $J=8.0 \mathrm{~Hz}, 1 \mathrm{H}, \operatorname{ArH}), 9.680(\mathrm{~s}, 1 \mathrm{H}$, ArNH), 10.624 (s, 1H, CONHCO); ESI-FTICR-MS calcd for $\mathrm{C}_{20} \mathrm{H}_{21} \mathrm{~F}_{2} \mathrm{IN}_{3} \mathrm{O}_{3} \mathrm{~S}[\mathrm{M}+\mathrm{H}]^{+}:$548.0316, found 548.0303.

$N^{2}$-(2-甲基-1-甲硫基-2-丙基)- $N^{1}$-(2-甲基苯基硫艮 基)-3-硝基邻苯二甲酰胺(7g): 浅黄色固体, 产率 $10 \%$. m.p. 101 102 ${ }^{\circ} \mathrm{C} ;{ }^{1} \mathrm{H}$ NMR $\left(400 \mathrm{~Hz}, \mathrm{CDCl}_{3}\right) \delta: 1.587$ [s, $\left.6 \mathrm{H}, \mathrm{C}\left(\mathrm{CH}_{3}\right)_{2}\right], 2.158\left(\mathrm{~s}, 3 \mathrm{H}, \mathrm{SCH}_{3}\right), 2.372\left(\mathrm{~s}, 3 \mathrm{H}, \mathrm{ArCH}_{3}\right)$, $2.995\left(\mathrm{~s}, 2 \mathrm{H}, \mathrm{SCH}_{2}\right), 6.270\left[\mathrm{~s}, 1 \mathrm{H}, \mathrm{CONHC}\left(\mathrm{CH}_{3}\right)_{2}\right], 7.281$ (t, $J=2.4 \mathrm{~Hz}, 1 \mathrm{H}, \mathrm{ArH}), 7.304(\mathrm{~d}, J=6.4 \mathrm{~Hz}, 1 \mathrm{H}, \mathrm{ArH})$, 7.557 (d, $J=3.2 \mathrm{~Hz}, 1 \mathrm{H}, \mathrm{ArH}), 7.706$ (t, $J=8.4 \mathrm{~Hz}, 1 \mathrm{H}$, ArH), 8.041 (d, $J=7.6 \mathrm{~Hz}, 1 \mathrm{H}, \mathrm{ArH}), 8.283$ (d, $J=8.4 \mathrm{~Hz}$, 1H, ArH), 10.040 (s, 1H, ArNH), 11.940 (s, 1H, CONHCS); ESI-FTICR-MS calcd for $\mathrm{C}_{21} \mathrm{H}_{24} \mathrm{~N}_{4} \mathrm{O}_{4} \mathrm{~S}_{2} \mathrm{Na}$ $[\mathrm{M}+\mathrm{Na}]^{+}:$: 483.1137, found 483.1132.

\subsection{2 目标化合物 8 的合成}

将化合物 7 (0.5 mmol)溶解到 $15 \mathrm{~mL}$ 四氢呋喃 (THF)中, 在室温下加入间氯过氧苯甲酸 $(1 \mathrm{mmol})$. 室温 搅拌, 点板跟踪反应. 待反应结束后, 将溶剂旋干, 得 到的固体用饱和 $\mathrm{Na}_{2} \mathrm{SO}_{3}$ 溶液, 饱和 $\mathrm{NaHCO}_{3}$ 溶液洗涤, 并用乙酸乙酯萃取. 合并有机相, 脱去溶剂, 所得固体 经柱层析得到化合物 8 .

$N^{2}$-(2-甲基-1-甲磺酰基-2-丙基)- $N^{1}$-苯嫝基-3-硝基 邻苯二甲酰胺 $(8 a)$ : 白色固体, 产率 72\%. m.p. 200 $201{ }^{\circ} \mathrm{C}$; ${ }^{1} \mathrm{H}$ NMR $\left(400 \mathrm{~Hz}, \mathrm{CDCl}_{3}\right) \delta: 1.532[\mathrm{~s}, 6 \mathrm{H}$, $\left.\mathrm{C}\left(\mathrm{CH}_{3}\right)_{2}\right], 3.038$ (s, $\left.3 \mathrm{H}, \mathrm{SCH}_{3}\right), 3.702\left(\mathrm{~s}, 2 \mathrm{H}, \mathrm{CH}_{2} \mathrm{~S}\right), 7.121$ (t, $J=8.0 \mathrm{~Hz}, 1 \mathrm{H}, \mathrm{ArH}), 7.362(\mathrm{t}, J=7.2 \mathrm{~Hz}, 2 \mathrm{H}, \mathrm{ArH})$, $7.556(\mathrm{~d}, J=8.0 \mathrm{~Hz}, 2 \mathrm{H}, \mathrm{ArH}), 7.773(\mathrm{t}, J=7.6 \mathrm{~Hz}, 1 \mathrm{H}$, ArH), 8.027 (d, $J=8.0 \mathrm{~Hz}, 1 \mathrm{H}, \mathrm{ArH}), 8.239$ (d, $J=8.0 \mathrm{~Hz}$, $1 \mathrm{H}, \mathrm{ArH}), 8.656\left[\mathrm{~s}, 1 \mathrm{H}, \mathrm{CONHC}\left(\mathrm{CH}_{3}\right)_{2}\right], 10.446(\mathrm{~s}, 1 \mathrm{H}$, ArNH), 11.116 (s, 1H, CONHCO); ESI-FTICR-MS calcd for $\mathrm{C}_{20} \mathrm{H}_{23} \mathrm{~N}_{4} \mathrm{O}_{7} \mathrm{~S}[\mathrm{M}+\mathrm{H}]^{+}:$463.1287, found 463.1279.
$N^{2}$-(2-甲基-1-甲磺酰基-2-丙基)- $N^{1}$ - 苯嫝基-3-碘邻 苯二甲酰胺 $(\mathbf{8 b})$ : 白色固体, 产率 $80 \%$. m.p. 162 $164{ }^{\circ} \mathrm{C} ;{ }^{1} \mathrm{H}$ NMR $\left(400 \mathrm{~Hz}, \mathrm{CDCl}_{3}\right) \delta:{ }^{1} \mathrm{H} \mathrm{NMR}(400 \mathrm{~Hz}$, $\left.\mathrm{CDCl}_{3}\right) \delta: 1.739\left[\mathrm{~s}, 6 \mathrm{H}, \mathrm{C}\left(\mathrm{CH}_{3}\right)_{2}\right], 2.692\left(\mathrm{~s}, 3 \mathrm{H}, \mathrm{SCH}_{3}\right)$, $3.680\left(\mathrm{~s}, 2 \mathrm{H}, \mathrm{CH}_{2} \mathrm{~S}\right), 7.010$ (t, $\left.J=8.0 \mathrm{~Hz}, 1 \mathrm{H}, \mathrm{ArH}\right), 7.099$ (m, 1H, ArH), 7.284 (d, J=1.6 Hz, 2H, ArH), 7.294 (s, $2 \mathrm{H}, \mathrm{ArH}), 7.482$ [s, $\left.1 \mathrm{H}, \mathrm{CONHC}\left(\mathrm{CH}_{3}\right)_{2}\right], 7.615$ (d, $J=8.0$ $\mathrm{Hz}, 1 \mathrm{H}, \mathrm{ArH}), 7.915$ (d, $J=8.0 \mathrm{~Hz}, 1 \mathrm{H}, \mathrm{ArH}), 9.422$ (s, $1 \mathrm{H}, \mathrm{ArNH}), 10.445$ (s, 1H, CONHCO); ESI-FTICR-MS calcd for $\mathrm{C}_{20} \mathrm{H}_{23} \mathrm{IN}_{3} \mathrm{O}_{5} \mathrm{~S}[\mathrm{M}+\mathrm{H}]^{+}:$544.0403, found 544.0391 .

$N^{2}$-(2-甲基-1-甲磺酰基-2-丙基)- $N^{1}$-(4-氯苯脲基)-3硝基邻苯二甲酰胺 $(\mathbf{8 c})$ : 白色固体, 产率 78\%. m.p. $222 \sim 224{ }^{\circ} \mathrm{C} ;{ }^{1} \mathrm{H}$ NMR $\left(400 \mathrm{~Hz}, \mathrm{DMSO}-d_{6}\right) \delta: 1.526[\mathrm{~s}$, $\left.6 \mathrm{H}, \mathrm{C}\left(\mathrm{CH}_{3}\right)_{2}\right], 3.043$ (s, $\left.3 \mathrm{H}, \mathrm{SCH}_{3}\right), 3.700\left(\mathrm{~s}, 2 \mathrm{H}, \mathrm{CH}_{2} \mathrm{~S}\right)$, 7.420 (d, $J=8.8 \mathrm{~Hz}, 2 \mathrm{H}, \mathrm{ArH}), 7.612(\mathrm{~d}, J=8.8 \mathrm{~Hz}, 2 \mathrm{H}$, ArH), $7.776(\mathrm{t}, J=8.0 \mathrm{~Hz}, 1 \mathrm{H}, \mathrm{ArH}), 8.029$ (d, $J=7.6 \mathrm{~Hz}$, $1 \mathrm{H}, \mathrm{ArH}), 8.247$ (d, $J=8.4 \mathrm{~Hz}, 1 \mathrm{H}, \mathrm{ArH}), 8.675[\mathrm{~s}, 1 \mathrm{H}$, $\left.\mathrm{CONHC}\left(\mathrm{CH}_{3}\right)_{2}\right], 10.494(\mathrm{~s}, 1 \mathrm{H}, \mathrm{ArNH}), 11.272(\mathrm{~s}, 1 \mathrm{H}$, CONHCO); ESI-FTICR-MS calcd for $\mathrm{C}_{20} \mathrm{H}_{22} \mathrm{ClN}_{4} \mathrm{O}_{7} \mathrm{~S}$ $[\mathrm{M}+\mathrm{H}]^{+}:$497.0898, found 497.0888.

$N^{2}$-(2-甲基-1-甲磺酰基-2-丙基)- $N^{1}$-(4-氯苯脲基)-3碘邻苯二甲酰胺 $(8 d)$ : 白色固体, 产率 66\%. m.p. 164 $165{ }^{\circ} \mathrm{C} ;{ }^{1} \mathrm{H}$ NMR $\left(400 \mathrm{~Hz}, \mathrm{CDCl}_{3}\right) \delta: 1.585[\mathrm{~s}, 6 \mathrm{H}$, $\left.\mathrm{C}\left(\mathrm{CH}_{3}\right)_{2}\right], 3.038\left(\mathrm{~s}, 3 \mathrm{H}, \mathrm{SCH}_{3}\right), 3.748\left(\mathrm{~s}, 2 \mathrm{H}, \mathrm{CH}_{2} \mathrm{~S}\right), 7.258$ (t, $J=8.8 \mathrm{~Hz}, 1 \mathrm{H}, \mathrm{ArH}), 7.405(\mathrm{~d}, J=8.0 \mathrm{~Hz}, 2 \mathrm{H}, \mathrm{ArH})$, 7.605 (d, $J=8.4 \mathrm{~Hz}, 2 \mathrm{H}, \mathrm{ArH}), 7.715$ (d, $J=9.2 \mathrm{~Hz}, 1 \mathrm{H}$, ArH), $8.049(\mathrm{~d}, J=8.0 \mathrm{~Hz}, 1 \mathrm{H}, \operatorname{ArH}), 8.410(\mathrm{~s}, 1 \mathrm{H}$, $\left.\mathrm{CONHC}\left(\mathrm{CH}_{3}\right)_{2}\right), 10.578$ (s, 1H, ArNH), 10.915 (s, 1H, CONHCO); ESI-FTICR-MS calcd for $\mathrm{C}_{20} \mathrm{H}_{22} \mathrm{CIIN}_{3} \mathrm{O}_{5} \mathrm{~S}$ $[\mathrm{M}+\mathrm{H}]^{+}:$578.0013, found 577.9999.

$N^{2}$-(2-甲基-1-甲磺酰基-2-丙基)- $N^{1}$-(4-氟苯脲基)-3碘邻苯二甲酰胺 $(\mathbf{8 e})$ : 白色固体, 产率 $78 \%$. m.p. 94 $95{ }^{\circ} \mathrm{C} ;{ }^{1} \mathrm{H}$ NMR $\left(400 \mathrm{~Hz}\right.$, DMSO- $\left.d_{6}\right) \delta: 1.585[\mathrm{~s}, 6 \mathrm{H}$, $\left.\mathrm{C}\left(\mathrm{CH}_{3}\right)_{2}\right], 3.038$ (s, 3H, $\left.\mathrm{SCH}_{3}\right), 3.746$ (s, $\left.2 \mathrm{H}, \mathrm{CH}_{2} \mathrm{~S}\right), 7.199$ (t, $J=8.9 \mathrm{~Hz}, 2 \mathrm{H}, \mathrm{ArH}), 7.262(\mathrm{t}, J=7.8 \mathrm{~Hz}, 1 \mathrm{H}, \mathrm{ArH})$, $7.567 \sim 7.602(\mathrm{~m}, 2 \mathrm{H}, \mathrm{ArH}), 7.708(\mathrm{~d}, J=8.0 \mathrm{~Hz}, 1 \mathrm{H}$, ArH), 8.049 (d, $J=8.8 \mathrm{~Hz}, 1 \mathrm{H}, \mathrm{ArH}), 8.431(\mathrm{~s}, 1 \mathrm{H}$, $\left.\mathrm{CONHC}\left(\mathrm{CH}_{3}\right)_{2}\right), 10.507$ (s, 1H, ArNH), $10.902(\mathrm{~s}, 1 \mathrm{H}$, CONHCO); ESI-FTICR-MS calcd for $\mathrm{C}_{20} \mathrm{H}_{22} \mathrm{FIN}_{3} \mathrm{O}_{5} \mathrm{~S}$ $[\mathrm{M}+\mathrm{H}]^{+}:$562.0309, found 562.0298.

$N^{2}$-(2-甲基-1-甲磺酰基-2-丙基)- $N^{1}$-(3,4-二氯苯脲 基)-3-碘邻苯二甲酰胺(8f): 白色固体, 产率 70\%. m.p. $154 \sim 155{ }^{\circ} \mathrm{C} ;{ }^{1} \mathrm{H}$ NMR $\left(400 \mathrm{~Hz}, \mathrm{CDCl}_{3}\right) \delta: 1.762[\mathrm{~s}, 6 \mathrm{H}$, $\left.\mathrm{C}\left(\mathrm{CH}_{3}\right)_{2}\right], 2.784$ (s, 3H, $\left.\mathrm{SCH}_{3}\right), 3.698$ (s, $\left.2 \mathrm{H}, \mathrm{CH}_{2} \mathrm{~S}\right), 6.972$ 
$(\mathrm{d}, J=9.0 \mathrm{~Hz}, 1 \mathrm{H}, \mathrm{ArH}), 7.017\left[\mathrm{~s}, 1 \mathrm{H}, \mathrm{CONHC}\left(\mathrm{CH}_{3}\right)_{2}\right]$, 7.075 (d, $J=8.9 \mathrm{~Hz}, 1 \mathrm{H}, \operatorname{ArH}), 7.115$ (t, $J=6.8 \mathrm{~Hz}, 1 \mathrm{H}$, ArH), 7.435 (ddd, $J=11.9,7.1,2.4 \mathrm{~Hz}, 1 \mathrm{H}, \mathrm{ArH}), 7.679$ (d, $J=7.6 \mathrm{~Hz}, 1 \mathrm{H}, \mathrm{ArH}), 7.983$ (d, $J=7.8 \mathrm{~Hz}, 1 \mathrm{H}, \mathrm{ArH})$, $9.414(\mathrm{~s}, 1 \mathrm{H}, \mathrm{ArNH}), 10.563$ (s, 1H, CONHCO). ESI-FTICR-MS calcd for $\mathrm{C}_{20} \mathrm{H}_{21} \mathrm{~F}_{2} \mathrm{IN}_{3} \mathrm{O}_{5} \mathrm{~S}[\mathrm{M}+\mathrm{H}]^{+}$: 580.0215 , found 580.0200 .

\subsection{3 目标化合物 9 的合成}

将化合物 7 (1 mmol)溶解到 $15 \mathrm{~mL}$ 四氢呋喃(THF) 中, 在冰浴下依次加入醋酸碘代苯 $(1.2 \mathrm{mmol})$ 、氰氨(1.5 $\mathrm{mmol})$. 冰浴下搅拌 $30 \mathrm{~min}$, 然后升温至室温摚拌, 点 板跟踪反应. 待反应结束后, 将溶剂旋干, 得到的固体 用 $1 \mathrm{~mol} / \mathrm{L}$ 稀盐酸、饱和 $\mathrm{NaHCO}_{3}$ 溶液、饱和 $\mathrm{NaCl}$ 溶 液洗涤, 并用乙酸乙酯萃取. 合并有机相, 脱去溶剂, 所得固体经柱层析得到化合物 9.

$N^{2}$-[2- 甲基-1-( $S$-( $N$ - 亚甲基氰氨 ) 甲硫基 )-2-丙 基]- $N^{1}$-苯嫝基-3-硝基邻苯二甲酰胺 $(\mathbf{9 a})$ : 白色固体, 产 率 75\%. m.p. $191 \sim 192{ }^{\circ} \mathrm{C} ;{ }^{1} \mathrm{H}$ NMR $\left(400 \mathrm{~Hz}, \mathrm{CDCl}_{3}\right) \delta$ : $1.529\left[\mathrm{~s}, 3 \mathrm{H}, \mathrm{C}\left(\mathrm{CH}_{3}\right)_{2}\right], 1.607\left[\mathrm{~s}, 3 \mathrm{H}, \mathrm{C}\left(\mathrm{CH}_{3}\right)_{2}\right], 2.671(\mathrm{~s}$, $\left.3 \mathrm{H}, \mathrm{SCH}_{3}\right), 3.204$ (d, $\left.J=12 \mathrm{~Hz}, 1 \mathrm{H}, \mathrm{CH}_{2} \mathrm{~S}\right), 3.056$ (d, $J=$ $11.6 \mathrm{~Hz}, 1 \mathrm{H}, \mathrm{CH}_{2} \mathrm{~S}$ ), 7.169 (t, $\left.J=7.6 \mathrm{~Hz}, 1 \mathrm{H}, \mathrm{ArH}\right), 7.329$ (t, $J=8.0 \mathrm{~Hz}, 2 \mathrm{H}, \mathrm{ArH}), 7.402$ (d, $J=7.6 \mathrm{~Hz}, 2 \mathrm{H}, \mathrm{ArH})$, $7.590\left[\mathrm{~s}, 1 \mathrm{H}, \mathrm{CONHC}\left(\mathrm{CH}_{3}\right)_{2}\right], 7.657(\mathrm{t}, J=8.0 \mathrm{~Hz}, 1 \mathrm{H}$, $\operatorname{ArH}), 7.956$ (d, $J=8.0 \mathrm{~Hz}, 1 \mathrm{H}, \operatorname{ArH}), 8.250$ (d, $J=8.0 \mathrm{~Hz}$, 1H, ArH), 10.114 (s, 1H, ArNH), 10.394 (s, 1H, CONH$\mathrm{CO}$ ). ESI-FTICR-MS calcd for $\mathrm{C}_{21} \mathrm{H}_{23} \mathrm{~N}_{6} \mathrm{O}_{5} \mathrm{~S}[\mathrm{M}+\mathrm{H}]^{+}$: 471.1451, found 471.1442.

$N^{2}$-[2- 甲基 -1-( $S$-( $N$ - 亚甲基氰氨) 甲硫基)-2-丙 基]- $N^{1}$-苯嫝基-3-碘邻苯二甲酰胺 $(9 \mathbf{b})$ : 白色固体, 产率 $81 \%$. m.p. $165 \sim 166{ }^{\circ} \mathrm{C}$; ${ }^{1} \mathrm{H}$ NMR $\left(400 \mathrm{~Hz}, \mathrm{CDCl}_{3}\right) \delta$ : $1.511\left(\mathrm{~d}, J=13.6 \mathrm{~Hz}, 6 \mathrm{H}, \mathrm{C}\left(\mathrm{CH}_{3}\right)_{2}\right), 2.841$ (s, 3H, $\left.\mathrm{SCH}_{3}\right)$, $3.897\left(\mathrm{~d}, J=13.6 \mathrm{~Hz}, 1 \mathrm{H}, \mathrm{CH}_{2} \mathrm{~S}\right), 4.025$ (dd, $J=6.8,14$ $\mathrm{Hz}, 2 \mathrm{H}, \mathrm{CH}_{2} \mathrm{~S}$ ), 7.116 (t, J=7.2 Hz, 1H, ArH), 7.266 (t, $J=8.0 \mathrm{~Hz}, 1 \mathrm{H}, \mathrm{ArH}), 7.357$ (t, $J=7.6 \mathrm{~Hz}, 2 \mathrm{H}, \mathrm{ArH}), 7.559$ (d, $J=7.6 \mathrm{~Hz}, 2 \mathrm{H}, \mathrm{ArH}), 7.725$ (d, $J=7.6 \mathrm{~Hz}, 1 \mathrm{H}, \mathrm{ArH})$, $8.054(\mathrm{~d}, J=8.0 \mathrm{~Hz}, 1 \mathrm{H}, \mathrm{ArH}), 8.633$ [s, 1H, CONH$\mathrm{C}\left(\mathrm{CH}_{3}\right)_{2}$ ], 10.430 (s, 1H, ArNH), 10.945 (s, 1H, CONHCO). ESI-FTICR-MS calcd for $\mathrm{C}_{21} \mathrm{H}_{23} \mathrm{IN}_{5} \mathrm{O}_{3} \mathrm{~S}[\mathrm{M}+\mathrm{H}]^{+}$: 552.0566, found 552.0556.

$N^{2}$-[2-甲基-1-( $S$-( $N$ - 亚甲基氰氨) 甲硫基)-2-丙 基]- $N^{1}$-(4-氯苯脲基)-3-硝基邻苯二甲酰胺(9c)：白色固 体, 产率 71\%. m.p. 208 209 ${ }^{\circ} \mathrm{C} ;{ }^{1} \mathrm{H}$ NMR $(400 \mathrm{~Hz}$, DMSO) $\delta: 1.453\left[\mathrm{~d}, J=19.2 \mathrm{~Hz}, 6 \mathrm{H}, \mathrm{C}\left(\mathrm{CH}_{3}\right)_{2}\right], 2.852$ (s, $3 \mathrm{H}, \mathrm{SCH}_{3}$ ), 3.343 (d, J=17.2 Hz, 1H, $\mathrm{CH}_{2} \mathrm{~S}$ ), 3.864 (d, $\left.J=13.6 \mathrm{~Hz}, 1 \mathrm{H}, \mathrm{CH}_{2} \mathrm{~S}\right), 7.421$ (d, $\left.J=8.8 \mathrm{~Hz}, 2 \mathrm{H}, \mathrm{ArH}\right)$, $7.632(\mathrm{~d}, J=8.8 \mathrm{~Hz}, 2 \mathrm{H}, \operatorname{ArH}), 7.790$ (t, $J=8.0 \mathrm{~Hz}, 1 \mathrm{H}$, ArH), 8.047 (d, $J=8.0 \mathrm{~Hz}, 1 \mathrm{H}, \mathrm{ArH}), 8.259$ (d, $J=8.4 \mathrm{~Hz}$, $1 \mathrm{H}, \mathrm{ArH}), 8.881$ [s, 1H, CONHC( $\left.\left(\mathrm{CH}_{3}\right)_{2}\right], 10.394(\mathrm{~s}, 1 \mathrm{H}$, ArNH), 11.334 (s, 1H, CONHCO); ESI-FTICR-MS calcd for $\mathrm{C}_{21} \mathrm{H}_{22} \mathrm{ClN}_{6} \mathrm{O}_{5} \mathrm{~S}[\mathrm{M}+\mathrm{H}]^{+}:$505.1061, found 505.1056.

$N^{2}$-[2-甲基-1-( $S$-( $N$ - 亚甲基氰氨) 甲硫基)-2-丙 基]- $N^{1}$-(4-氯苯嫝基)-3-碘邻苯二甲酰胺(9d): 白色固体, 产率 $80 \%$. m.p. $153 \sim 154{ }^{\circ} \mathrm{C}$; ${ }^{1} \mathrm{H}$ NMR $(400 \mathrm{~Hz}$, DMSO- $\left.d_{6}\right) \delta: 1.518\left[\mathrm{~d}, J=13.6 \mathrm{~Hz}, 6 \mathrm{H}, \mathrm{C}\left(\mathrm{CH}_{3}\right)_{2}\right], 2.851$ (s, 3H, $\mathrm{SCH}_{3}$ ), 3.362 (d, J=13.6 Hz, 1H, $\left.\mathrm{CH}_{2} \mathrm{~S}\right), 3.898$ (d, $\left.J=13.6 \mathrm{~Hz}, 1 \mathrm{H}, \mathrm{CH}_{2} \mathrm{~S}\right), 7.276(\mathrm{t}, J=7.6 \mathrm{~Hz}, 1 \mathrm{H}, \mathrm{ArH})$, $7.416(\mathrm{~d}, J=8.4 \mathrm{~Hz}, 2 \mathrm{H}, \operatorname{ArH}), 7.620$ (d, $J=8.8 \mathrm{~Hz}, 2 \mathrm{H}$, ArH), 7.729 (d, $J=8.0 \mathrm{~Hz}, 1 \mathrm{H}, \mathrm{ArH}), 8.064$ (d, $J=8.0 \mathrm{~Hz}$, $1 \mathrm{H}, \mathrm{ArH}), 8.635\left[\mathrm{~s}, 1 \mathrm{H}, \mathrm{CONHC}\left(\mathrm{CH}_{3}\right)_{2}\right], 10.479$ (s, $1 \mathrm{H}$, ArNH), 11.006 (s, 1H, CONHCO); ESI-FTICR-MS calcd for $\mathrm{C}_{21} \mathrm{H}_{22} \mathrm{ClIN}_{5} \mathrm{O}_{3} \mathrm{~S}[\mathrm{M}+\mathrm{H}]^{+}:$586.0177, found 586.0162.

\section{7 目标化合物的生物活性测试}

采用文献[33]的方法对东方粘虫(Mythimnaseparate Walker)进行了杀虫活性测试. 采用菌丝生长速率法 [34, 35 ], 在 $50 \mathrm{mg} / \mathrm{L}$ 的浓度下, 对番茄早疫、小麦赤霉、辣 椒疫霉、油菜菌核、水稻纹枯、黄瓜枯萎、花生褐斑、 苹果轮纹、小麦纹枯、玉米小斑、西瓜炭疽、水稻恶苗 这 12 种常见病害进行了抑菌测试.

辅助材料 (Supporting Information) 所有目标产物 $7 \mathbf{a} \sim 7 \mathbf{g}, 8 \mathbf{a} \sim 8 \mathbf{f}, 9 \mathbf{a} \sim 9 \mathbf{d}$ 的氢谱核磁共振 $\left({ }^{1} \mathrm{H} \mathrm{NMR}\right)$ 和高 分辨质谱(HMRS)谱图. 这些材料可以免费从本刊网站 (http://sioc-journal.cn/)上下载.

\section{References}

[1] Pan, L.; Chen, Y. W.; Liu, Z.; Li, Y. H.; Li, Z. M. Chin. J. Org. Chem. 2013, 33, 542 (in Chinese).

(潘里, 陈有为, 刘卓, 李永红, 李正名, 有机化学, 2013, 33, 542.)

[2] Tong, J. Y.; Shi, Y. X.; Liu, X. H.; Sun, N. B.; Li, B. J. Chin. J. Org. Chem. 2012, 32, 2373 (in Chinese).

(童建顷, 石延霞, 刘幸海, 孙娜波, 李宝聚, 有机化学, 2012, 33, 2373.)

[3] Tan, C. X.; Shi, Y. X.; Weng, J. Q.; Liu, X. H.; Zhao, W. G.; Li, B. J. J. Heterocycl. Chem., 2014, 51, 690.

[4] Zhang, L. J.; Yang, M. Y.; Sun, Z. H.; Tan, C. X.; Weng, J. Q.; Wu, H. K.; Liu, X. H. Lett. Drug Des. Discovery 2014, 11, 1107.

[5] Ogawa, Y. Crit. Rev. Biochem. Mol. Biol. 1994, 29, 229.

[6] Dong, W. L.; Xu, J. Y.; Liu, X. H.; Zhao, W. G.; Li, Z. M.. Chin. J. Pest. Sci. 2008, 10, 178.

[7] Sattelle, D. B.; Cordova, D.; Cheek, T. R. Invertebr. Neurosci. 2008, 8,107 .

[8] Feng, Q.; Liu, Z. L.; Wang, M. Z.; Xiong, L. X.; Yu, S. J.; Li, Z. M. Chem. J. Chin. Univ. 2011, 32, 74.

[9] Chen, Y. W.; Li, Y. X.; Pan, L.; Liu, J. B. Wang, Y. Y.; Chen, W.; 
Xiong, L. X. Yang, N.; Song, H. B.; Li, Z. M. Bioorg. Med. Chem. 2014, 22, 6366 .

[10] Tohnishi, M.; Nakao, H.; Kohno, E.; Nishida, T.; Furuya, T.; Shimizu, T.; Seo, A.; Sakata, K.; Fujioka, S.; Kano, H. EP 919542, 1999 [Chem. Abstr. 1999, 131, 31808].

[11] Liu, C. L. Agrochemicals 2005, 44(11), 527 (in Chinese). (刘长令, 农药, 2005, 44(11), 527.)

[12] Li, Y.; Li, M.; Cai, B. S. Agrochemicals 2006, 45(10), 697 (in Chinese).

(李洋, 李丞, 柴宝山, 农药, 2006, 45(10), 697.)

[13] Chen, Y. W.; Wang, B. L.; Guo, Y. J.; Zhou, Y. Y.; Pan, L.; Xiong, L. X.; Yu, S. J.; Li, Z. M. Chem. Res. Chin. Univ. 2014, 30, 98.

[14] Zhang, X. L.; Li, Y. X., Ma, J. L.; Zhu, H. W.; Wang, B. L.; Mao, M. Z.; Xiong, L. X..; Li, Y. Q.; Li, Z. M. Bioorg. Med. Chem. 2014, $22,186$.

[15] Julius, J. J. Agric. Food. Chem. 1980, $28(l), 2$.

[16] Wellinga. J. Agric. Food. Chem. 1973, $21(2), 345$.

[17] Lin, J.; Yan, S. J.; Yang, L. J.; Li, J. F.; Liu, F. C. Chin. J. Org. Chem. 2005, 25, 304 (in Chinese).

(林军, 严胜骄, 杨丽娟, 李俊峰, 刘复初, 有机化学, 2005, 25, 304.)

[18] Lahm, G. P.; Selby, T. P.; Frendenberger, J. H.; Stevenson, T. M.; Myers, B. J.; Seburyamo, G.; Smith, B. K.; Flexner, L.; Clark, C. E.; Cordova, D.; Bioorg. Med. Chem. Lett. 2005, 15, 4898.

[19] Zhang, J. F.; Xu, J. Y.; Wang, B. L.; Li, Y. X.; Xiong, L. X.; Li, Y. Q.; Ma, Y.; Li, Z. M. J. Agric. Food Chem. 2012, 60, 7565.

[20] O'sullivan, A C.; Lutz, W. J.; AndreRobson, M. J. Smith, S. WO 2006024523, 2006 [Chem. Abstr. 2006, 144, 292427].

[21] Ikeda, E.; Maeda, K.; Furukawa, Y.; Takii, S. WO 2005030699 , 2005 [Chem. Abstr. 2005, 142, 373562].

[22] Liu, X. H.; Weng, J. Q.; Tan, C. X. J. Chem. 2013, 2013, 306361.
[23] Liu, X. H.; Sun, Z. H.; Yang, M. Y.; Tan, C. X.; Weng, J. Q.; Zhang, Y. G.; Ma, Y. Chem. Biol. Drug Des. 2014, 84, 342.

[24] Liu, X. H.; Weng, J. Q.; Tan, C. X.; Liu, H. J. Acta Crystallogr. 2012, E68, O493.

[25] Tong, J. Y.; Wu, H. K.; Sun, N. B.; Liu, X. H. Chin. J. Struct. Chem. 2013, 32, 607.

[26] Liu, X. H.; Xu, X. Y.; Tan, C. X.; Weng, J. Q.; Xin, J. H.; Chen, J. Pest Manage. Sci. 2015, 71(2), 292.

[27] Srikanth, L.; Usha, N.; Ramesh, J.; Raghunandan, N.; Venkateshwar, R. J.; Raj, M. K. Pharm. Chem. 2010, 2(4), 231.

[28] Sohail, S.; Naghmana, R.; Peter, G. J.; Muhammad, A.; Rizwan, H. Eur. J. Med. Chem. 2010, 45, 1323.

[29] Siddiqui, N.; Ahsan, W. Eur. J. Med. Chem. 2010, 45, 1536.

[30] Feng, M. L.; Li, Y. F.; Zhu, H. J.; Liang, Z.; Xi, B. B.; Ni, J. P. J. Agric. Food Chem. 2010, 58, 10999.

[31] Feng, M. L.; Li, Y. F.; Zhu, H. J.; Zhao, L.; Xi, B. B.; Ni, J. P. J. Agric. Food Chem. 2010, 58, 10999.

[32] Lin, T.; Zhao, D. J.; Mao, C. H.; Wu, D. X.; Yang, B. Fine Chem. Interm. 2010, 40, 29.

[33] Wang, B. L.; Ma, Y.; Xiong, L. X.; Li, Z. M. Chin. J. Chem. 2012, 30,815 .

[34] Chen, N. C. Bioassay Technology for Pestcides, Beijing Agricultural University Press, Beijing, 1991, p. 161 (in Chinese). (陈春年，农药生物技术测定，北京农业大学出版社，北京， 1991, p. 161.)

[35] Jiang, W. T.; Hu, F. Z.; Gu, H. Liu, C.; Wei, N. X.; Wan, L.; Ren, S. Z.; Wang, J. T.; Xu, F. B. Chin. J. Org. Chem. 2014, 34, 774 (in Chinese).

(姜文涛, 胡方中, 顾 翰, 刘传, 魏乃翔, 万蕾, 任士钊, 王俊 婷, 徐凤波, 有机化学, 2014, 34, 774.) 